\title{
Molecular Dynamics Simulation of High-Temperature Creep Behavior of Nickel Polycrystalline Nanopillars
}

\author{
Xiang $\mathrm{Xu}$ *, Peter Binkele, Wolfgang Verestek and Siegfried Schmauder
}

check for

updates

Citation: $\mathrm{Xu}, \mathrm{X}$.; Binkele, P.;

Verestek, W.; Schmauder, S. Molecular

Dynamics Simulation of

High-Temperature Creep Behavior of Nickel Polycrystalline Nanopillars.

Molecules 2021, 26, 2606.

https://doi.org/10.3390/molecules 26092606

Academic Editors: Tomasz Sadowski, Liviu Marsavina and Nikolaos Michailidis

Received: 8 March 2021

Accepted: 26 April 2021

Published: 29 April 2021

Publisher's Note: MDPI stays neutral with regard to jurisdictional claims in published maps and institutional affiliations.

Copyright: (c) 2021 by the authors. Licensee MDPI, Basel, Switzerland. This article is an open access article distributed under the terms and conditions of the Creative Commons Attribution (CC BY) license (https:// creativecommons.org/licenses/by/ $4.0 /)$.
Institute for Materials Testing, Materials Science and Strength of Materials, University of Stuttgart, Pfaffenwaldring 32, 70569 Stuttgart, Germany; peter.binkele@imwf.uni-stuttgart.de (P.B.); wolfgang.verestek@imwf.uni-stuttgart.de (W.V.); Siegfried.Schmauder@imwf.uni-stuttgart.de (S.S.)

* Correspondence: xiang.xu@imwf.uni-stuttgart.de

Abstract: As Nickel (Ni) is the base of important Ni-based superalloys for high-temperature applications, it is important to determine the creep behavior of its nano-polycrystals. The nano-tensile properties and creep behavior of nickel polycrystalline nanopillars are investigated employing molecular dynamics simulations under different temperatures, stresses, and grain sizes. The mechanisms behind the creep behavior are analyzed in detail by calculating the stress exponents, grain boundary exponents, and activation energies. The novel results in this work are summarized in a deformation mechanism map and are in good agreement with Ashby's experimental results for pure Ni. Through the deformation diagram, dislocation creep dominates the creep process when applying a high stress, while grain boundary sliding prevails at lower stress levels. These two mechanisms could also be coupled together for a low-stress but a high-temperature creep simulation. In this work, the dislocation creep is clearly observed and discussed in detail. Through analyzing the activation energies, vacancy diffusion begins to play an important role in enhancing the grain boundary creep in the creep process when the temperature is above $1000 \mathrm{~K}$.

Keywords: polycrystalline nanopillars; molecular dynamics method; creep mechanisms; dislocation creep; grain boundary sliding; deformation map

\section{Introduction}

Nanocrystalline (NC) metals possess many different mechanical behaviors in comparison with traditional coarse grained metals, e.g., the Hall-Petch effect and the inverse Hall-Petch effect, which have already been studied by several researchers [1-4]. For application at a high-temperature condition, the creep behavior is the most important one that should be carefully researched and thoroughly understood. The creep deformation in polycrystalline metals is well described by the Bird-Dorn-Mukherjee relation [5], given below as Equation (1).

$$
\dot{\varepsilon}=\frac{A D_{0} G b}{k_{\mathrm{b}} T}\left(\frac{b}{d}\right)^{p}\left(\frac{\sigma}{G}\right)^{n} \exp \left(-\frac{\Delta Q}{k_{\mathrm{b}} T}\right)
$$

In this equation, $A$ is a dimensionless constant, $D_{0}$ is the diffusion constant (frequency factor), and $b$ is the Burgers vector. $G, k_{\mathrm{b}}$, and $\Delta Q$ are the shear modulus, the Boltzmann constant, and the activation energy for a thermally activated process, respectively. $p$ represents the exponent of the grain size $d$ and $n$ the exponent of the applied stress $\sigma$. T is the temperature. With varying the temperature $T$, the applied stress $\sigma$, and the grain size $d$ in a creep simulation, different creep behavior can be observed.

On the nanoscale, the diffusion process and the grain boundaries influence the creep mechanisms in a significant way, therefore many studies on this topic have already been published [6-12]. The exponent pair $(n, p)$ for stress and grain size was used to determine the creep mechanisms. For example, $n=1, p=2$ was characterized as Nabarro-Herring creep (lattice diffusion) [10], and $n=1, p=3$ is Coble creep [6]. When $n>3$, the plastic 
deformation mechanism is dislocation creep, i.e., the power-law creep [13-15]. Moreover, Ashby introduced deformation maps that display the fields of stress and temperature in which a particular mechanism of plastic flow is dominant [16].

The development of molecular dynamics simulations provides a way to study the creep behavior of nanocrystalline materials. Swygenhoven et al. reported that the grain boundary sliding, motion, and orientation were responsible for the low-temperature high-load plastic deformation of the Ni nanophase [17]. The publications [18-20] approached creep behavior from the diffusion mechanism, the bending creep behavior, and the influence of porosity and voids on the creep behavior, respectively. Yamakov et al. studied the deformation mechanism, the nucleation and propagation of dislocations, the grain boundary diffusion, and the sliding of grain boundaries through MD simulations [21-25]. Moreover, addition of alloying elements also influences the creep behavior [26].

Because of the limitations of computer abilities, the grain size is restricted by the number of atoms inside the NC model. In previous works [12-14,27], the investigated grain sizes were not bigger than $16 \mathrm{~nm}$, which led to a small free space for intra-grain dislocation movements. Hence, the discovered creep mechanisms were mostly limited to diffusion creep, grain boundary sliding, and dislocation nucleation.

Especially for Ni nanocrystals, Nie et al. [12] studied the creep behavior of NC Ni with consideration on temperatures, grain sizes, and stresses. The grain sizes in their work ranged from 2.8 to $5.6 \mathrm{~nm}$. It was found that with increasing the temperature and stress level, the creep mechanisms changed from lattice diffusion and grain boundary sliding to grain boundary diffusion and then to dislocation nucleation. However, due to the limitation of grain sizes, the most important dislocation propagation and interactions were not studied.

This research took the temperature $T$, the applied stress $\sigma$, and the grain size $d$ as variables and studied their influence on the creep behavior of Ni polycrystalline nanopillars. We chose grain sizes between $20-30 \mathrm{~nm}$ and discovered that the dislocations can interact with other dislocations or grain boundaries. Creep mechanisms were determined through stress exponents $n$ and grain size exponents $p$. Corresponding mechanisms were also verified with the atomistic visualization of configurations dumped during the creep process.

\section{Simulation Method and Process}

Considering the accessible computer resources, our models contain approximately 10,000,000 atoms. All the models were first set up through the software Atomsk [28], using the implemented Voronoi method with random orientations and positions of crystalline seeds, which results in a nearly isotropic property for the model. After that, the models were simulated through the Large-scale Atomic/Molecular Massively Parallel Simulator (LAMMPS) [29]. An embedded atom method (EAM) potential Fe-Ni-Cu by Bonny [30] was used, in which the potential for Ni was adopted from Voter and Chen [31]. The elastic properties of $\mathrm{Ni}$, computed with this potential, are summarized in Table 1. The atomistic visualization was carried out through the Open Visualization Tool (OVITO) [32].

Table 1. The modulus of pure Ni at different temperatures.

\begin{tabular}{ccccc}
\hline $\begin{array}{c}\text { Temperature } \\
\boldsymbol{T}[\mathrm{K}]\end{array}$ & $\begin{array}{c}\text { Bulk Modulus } \\
\text { K [GPa] }\end{array}$ & $\begin{array}{c}\text { Poisson's Ratio } \\
\boldsymbol{\mu}[-]\end{array}$ & $\begin{array}{c}\text { Young's Modulus } \\
\boldsymbol{E}[\mathrm{GPa}]\end{array}$ & $\begin{array}{c}\text { Shear Modulus } \\
\boldsymbol{G} \text { [GPa] }\end{array}$ \\
\hline 500 & 162.754 & 0.379 & 223.330 & 95.427 \\
\hline 800 & 150.985 & 0.379 & 207.814 & 88.310 \\
\hline 1200 & 135.164 & 0.381 & 189.529 & 77.88 \\
\hline
\end{tabular}

\subsection{Main Variables}

In this study, the main variables were the mean grain size $d$ of the model, the temperature $T$, and the applied stress $\sigma$. The initial size of simulation boxes was $50 \times 50 \times$ 
$50 \mathrm{~nm}^{3}$. The number of grains varied from 10 to 30 in five models, see Figure 1 . Through approximating grains as spheres, the corresponding averaged diameter $\bar{d}$ can be calculated by $\bar{d}=\sqrt[3]{3 V /(4 \pi N)}$, in which $V$ and $N$ are the volume of the model and the number of grains in the model, respectively. Table 2 shows the grain size of each model.

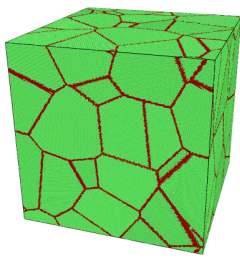

(a)

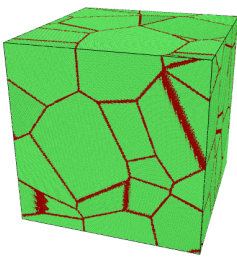

(b)

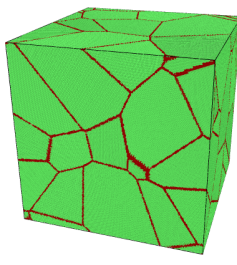

(c)

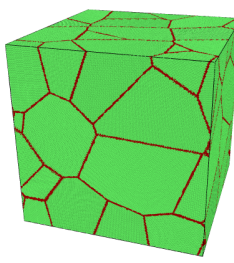

(d)

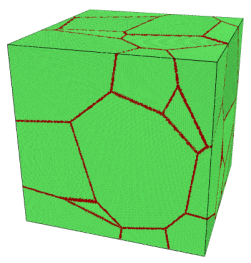

(e)

Figure 1. (a-e) Five models with different numbers of grains, named M1 to M5 for model 1 to model 5. These pictures are visualized by a polyhedral template matching (PTM) method [33] in OVITO (green: FCC; red: others as grain boundary). (a) M1: 30 grains, (b) M2: 25 grains, (c) M3: 20 grains, (d) M4: 15 grains, (e) M5: 10 grains.

Table 2. The averaged grain size $d$ of every model.

\begin{tabular}{ccc}
\hline Model & Number of Grains $\boldsymbol{N}$ & Averaged Grain Size $\boldsymbol{d}[\mathbf{n m}]$ \\
\hline M1 & 30 & 19.96 \\
\hline M2 & 25 & 21.22 \\
\hline M3 & 20 & 22.85 \\
\hline M4 & 15 & 25.15 \\
\hline M5 & 10 & 28.79 \\
\hline
\end{tabular}

The melting point $T_{\mathrm{m}}$ of pure Ni was simulated in this work as $1720 \mathrm{~K}$, which is close to the well-known value $1728 \mathrm{~K}$. The investigated temperatures are 500,800 , and $1200 \mathrm{~K}$ for all five models. Additionally, in order to obtain more detailed information about the influence of temperature and to analyze the activation energy, the model M1 was simulated at a finer temperature mesh from 500 to $1200 \mathrm{~K}$ for every $100 \mathrm{~K}$ increment.

All the applied stresses in the creep simulations were homologous to the strength $R_{\mathrm{m}}$. The applied stresses varied from $0.4 R_{\mathrm{m}}$ to $0.8 R_{\mathrm{m}}$.

\subsection{Main Process of Simulations}

The models were initially equilibrated at the selected temperatures, i.e., 500, 800, and $1200 \mathrm{~K}$, as an isotherm-isobar (NPT) ensemble. Then the nano-tensile simulations were performed. The nano-creep simulations were sequentially executed with the applied uniaxial stresses level determined by the tensile strength. The applied boundary conditions of nano-tensile and -creep simulations were periodic in the $x$-direction and shrink-wrapped (not periodic but encompassing all the atoms inside the surface) in the y-and z-directions.

\section{Results and Analysis of Simulations}

\subsection{Nano-Tensile Test Simulations}

In order to obtain strengths $\mathrm{R}_{\mathrm{m}}$ of all models M1-M5, nano-tensile tests at different temperatures were carried out prior to nano-creep simulations.

Figure 2a shows the stress-strain curves of M1 at different temperatures. At the beginning, it is a linear elastic region, and the slope of this section ( $\varepsilon$ to $0.5 \%$ ) is the Young's modulus, $221.25 \mathrm{GPa}$ at $500 \mathrm{~K}$. Compared with Table 1, we can see that the difference is very small. This indicates that the model is almost isotropic.

In Figure 2a an oscillation can be observed of the resulting tensile stress in the stressstrain curve with the frequency varying from 14 to $18 \mathrm{ps}$ (strain from 1.4 to $1.8 \%$ ). This 
oscillation is considered to be caused by the non-periodic boundary conditions in the yand z-directions in combination with the elastic pulse when starting the tensile simulation. Furthermore, the maximum amplitude at the first cycle at a strain of approximately $0.9 \%$ shows a higher stress at $1200 \mathrm{~K}$ than at $800 \mathrm{~K}$ and $500 \mathrm{~K}$, and indicates a temperature influence on this effect. Further analysis shows that the oscillation decays rapidly and therefore, the influence of the oscillation on the ultimate tensile strength is minor.

Figure $2 b, c$ display the temperature influence on the tensile strength of M1. The tensile strength of model M1 decreases from 3.08 to $2.25 \mathrm{GPa}$ as the temperature increases, which is due to the softening of the material at higher temperatures. Figure $2 \mathrm{c}$ demonstrates the relation between the grain size and the tensile strength $R_{\mathrm{m}}$. The tensile strength shows no significant dependence on the grain size in the investigated range. This suggests that the relevant grain sizes lie around the transition regime between the Hall-Petch and the reverse Hall-Petch effect.

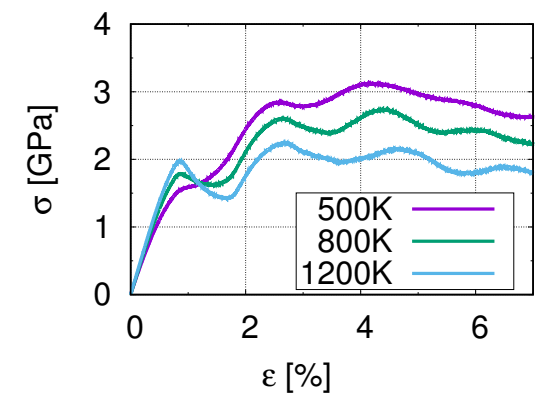

(a)

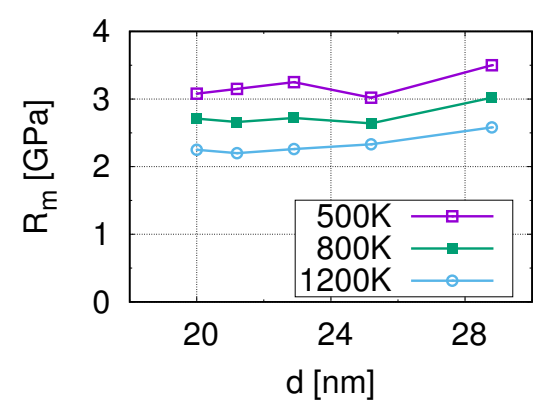

(c)

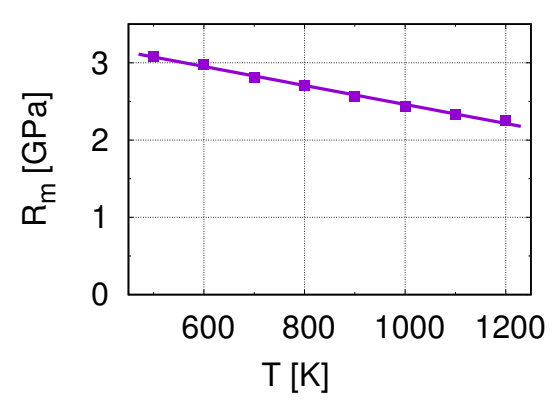

(b)

Figure 2. The results of nano-tensile simulations. (a) Stress-strain curves at the corresponding temperatures for M1. (b) The temperature dependence of the tensile strength $R_{\mathrm{m}}$. (c) The influence of the grain size on the tensile strength $R_{\mathrm{m}}$.

\subsection{Nano-Creep Simulations}

As shown in Figure 3a, the creep strain increases with the simulation time. At the beginning of the simulation, there are some fluctuations of the creep strain, because the stress was applied to the model within a short time interval. The second phase of the creep process is steady and linear, and the creep rate $\dot{\varepsilon}=\mathrm{d} \varepsilon / \mathrm{d} t$ in this phase is the minimum creep rate during the whole process. Under an applied stress $\sigma=0.7 R_{\mathrm{m}}$, the creep process of model M1 steps into the third phase at $200 \mathrm{ps}$. When the applied stress increases to $0.8 R_{\mathrm{m}}$, the second phase is very short and the creep process steps directly into the third phase. The second creep phase, which can be clearly observed in this work, plays the most important role during the creep process. Therefore, the simulation time around $500 \mathrm{ps}$ is sufficient to investigate the dominant creep mechanisms. 


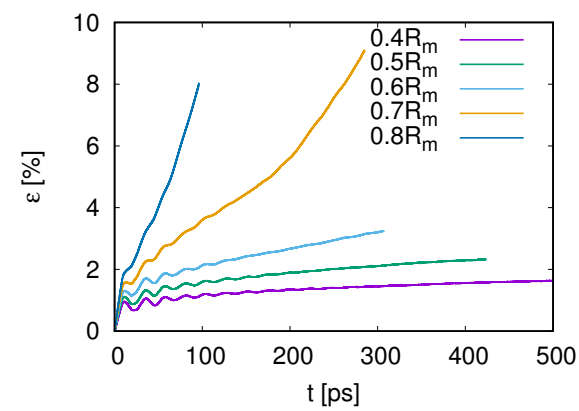

(a)

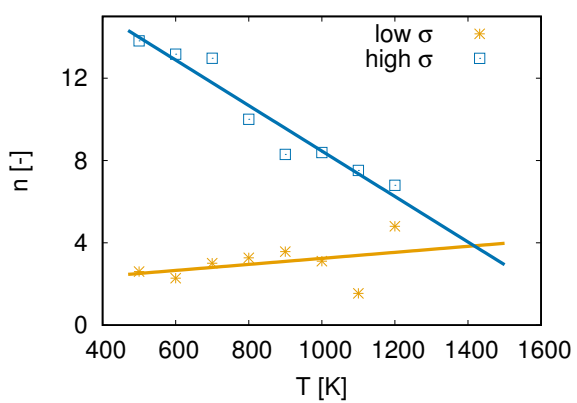

(c)

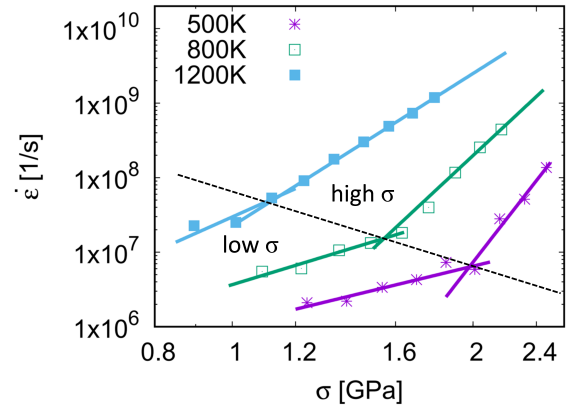

(b)

Figure 3. (a) Creep curves of model M1 for different stresses at $1000 \mathrm{~K}$. (b) The log-log scaling plot of creep rates $\dot{\varepsilon}$ over $\sigma$ at different temperatures. The data are split up into a low $\sigma$ region and a high $\sigma$ region and were fitted with different exponents $n$. (c) The relationship between the stress exponent $n$ and temperature.

The minimum creep rate $\dot{\varepsilon}$ is of great importance to the creep property. In Figure $3 b$, the relation of $\dot{\varepsilon}$ and $\sigma$ at different temperatures $T$ is revealed in a log-log scaling diagram. The higher the applied stress is, the faster the model creeps. Furthermore, it can also be seen from Figure $3 b$ that the creep rate $\dot{\varepsilon}$ increases with temperature.

According to the power-law relationship of the strain rate $\dot{\varepsilon}$ and the applied stress $\sigma$ from the Bird-Dorn-Mukherjee relation (Equation (1)), the stress exponent is expressed as $n=\partial \log \dot{\varepsilon} / \partial \log \sigma . n$ is the slope in the $\log -\log$ scaling plot of $\dot{\varepsilon}$ with $\sigma$. As displayed in Figure $3 \mathrm{~b}$, the relationship between $\log \dot{\varepsilon}$ and $\log \left(\sigma / R_{\mathrm{m}}\right)$ is not linear. Therefore the data were cut into two regions, a low $\sigma$ region and a high $\sigma$ one, and these calculated exponents are shown in Figure $3 c$ and in Table 3.

Table 3. The stress exponents $n$ for different stresses and temperatures.

\begin{tabular}{ccccccccc}
\hline & $\mathbf{5 0 0} \mathbf{K}$ & $\mathbf{6 0 0} \mathbf{K}$ & $\mathbf{7 0 0} \mathbf{K}$ & $\mathbf{8 0 0} \mathbf{K}$ & $\mathbf{9 0 0} \mathbf{K}$ & $\mathbf{1 0 0 0} \mathbf{K}$ & $\mathbf{1 1 0 0} \mathbf{K}$ & $\mathbf{1 2 0 0} \mathbf{K}$ \\
\hline low $\sigma$ & 2.6 & 2.3 & 3.0 & 3.3 & 3.6 & 3.1 & 1.5 & 4.8 \\
\hline high $\sigma$ & 13.8 & 13.2 & 13.0 & 10.0 & 8.3 & 8.4 & 7.5 & 6.8 \\
\hline
\end{tabular}

From Figure $3 \mathrm{c}$ and Table 3 , it is considered that when applying high stresses at a temperature between 500 and $1200 \mathrm{~K}$, the dominant mechanism is the power-law creep as is well-known as the dislocation creep because of stress exponents $n>3$.

For creep tests at low stresses, stress exponents are larger than 2 and increase with temperature. When $T<700 \mathrm{~K}$, the creep mechanism for low stresses is distinguished as grain boundary sliding as the stress exponent $2.6<n<3$ [12]. When the temperature is above $800 \mathrm{~K}$, the creep mechanism is supposed to be coupled by grain boundary sliding and dislocation nucleation and propagation.

The yellow line fitted with stress exponents of low $\sigma$ regions shows a deviation for the two points at 1100 and $1200 \mathrm{~K}$. This is due to fewer data points for fitting low stress 
exponents at a high temperature, e.g., 6 points at $500 \mathrm{~K}$ but 3 points at $1200 \mathrm{~K}$ in Figure $3 \mathrm{~b}$. However, the yellow line can still provide an approximate prediction of the stress exponent $n$ of the low $\sigma$ region for higher temperature conditions.

It is interesting to emphasize that the stress exponents of low- and high-stress parts have an intersection at around $(1400 \mathrm{~K}, n=4)$, as shown in Figure 3c. This means that the creep mechanism above $1400 \mathrm{~K}$ is independent of the stress ranging from $0.4 R_{\mathrm{m}}$ to $0.8 R_{\mathrm{m}}$, and the stress exponent $n=4$ represents that the grain boundary sliding is coupled with dislocation slip (see Section 4.3).

\subsection{Thermally Activated Mechanisms}

The Arrhenius equation was applied here to analyze the temperature influence on the creep rate. Figure 4 shows the data and fitted lines for $\ln \dot{\varepsilon}$ and $1 /\left(k_{\mathrm{b}} T\right)$, which are derived from the Arrhenius equation. The slope of every fit line is the free activation energy $\Delta G$ of the creep process.

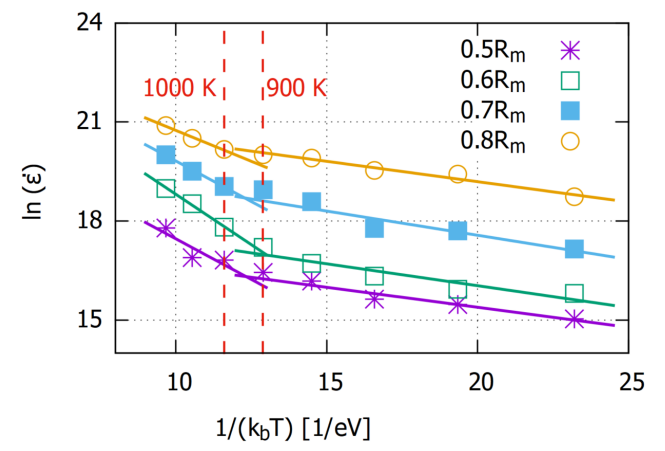

(a)

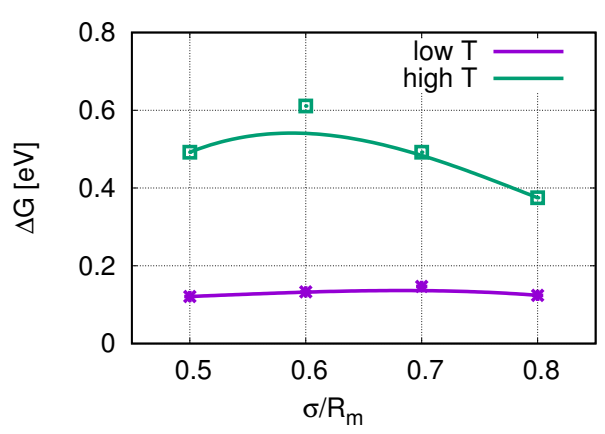

(b)

Figure 4. (a) The plot of $\ln \dot{\varepsilon}$ versus $1 /\left(k_{B} T\right)$, which is derived from the Arrhenius equation. (b) Corresponding activation energies.

From Figure $4 \mathrm{a}$, the creep rate at a certain stress can be divided into two different temperature ranges. The turning point is around $900 \sim 1000 \mathrm{~K}$ for all applied stresses. It is assumed to be the thermal activation for the vacancy diffusion as Aidhy et al. [34] reported that the vacancies are immobile up to $700 \mathrm{~K}$ and diffusing at $1200 \mathrm{~K}$. At high temperatures, the activation energy for the accelerated creep process is around $0.5 \mathrm{eV}$, which is slightly lower than the $0.8 \mathrm{eV}$ reported by Swygenhoven [18]. This might be caused by a couple mechanism with grain boundary diffusion or dislocation gliding.

\section{Discussion on Creep Mechanisms}

\subsection{Deformation Diagram for NC Ni}

Although the creep rate in the MD simulation is of around 8 orders of magnitude faster than in experimental results, which is the typical timescale and/or length scale problem for MD simulations, the simulation results reveal the mechanism of plastic deformations when we normalize the parameters to dimensionless.

In order to compare our work with experimental results, all plastic deformations were collected and a deformation diagram for NC Ni was drafted in this work, as shown in Figure 5. The applied stresses were normalized to the corresponding tensile strength $R_{\mathrm{m}}$ at each temperature. The green, blue and red regions were divided by certain $(\sigma, T)$ points as investigated in this work, which represent grain boundary sliding, dislocation creep, and their coupling, respectively. 


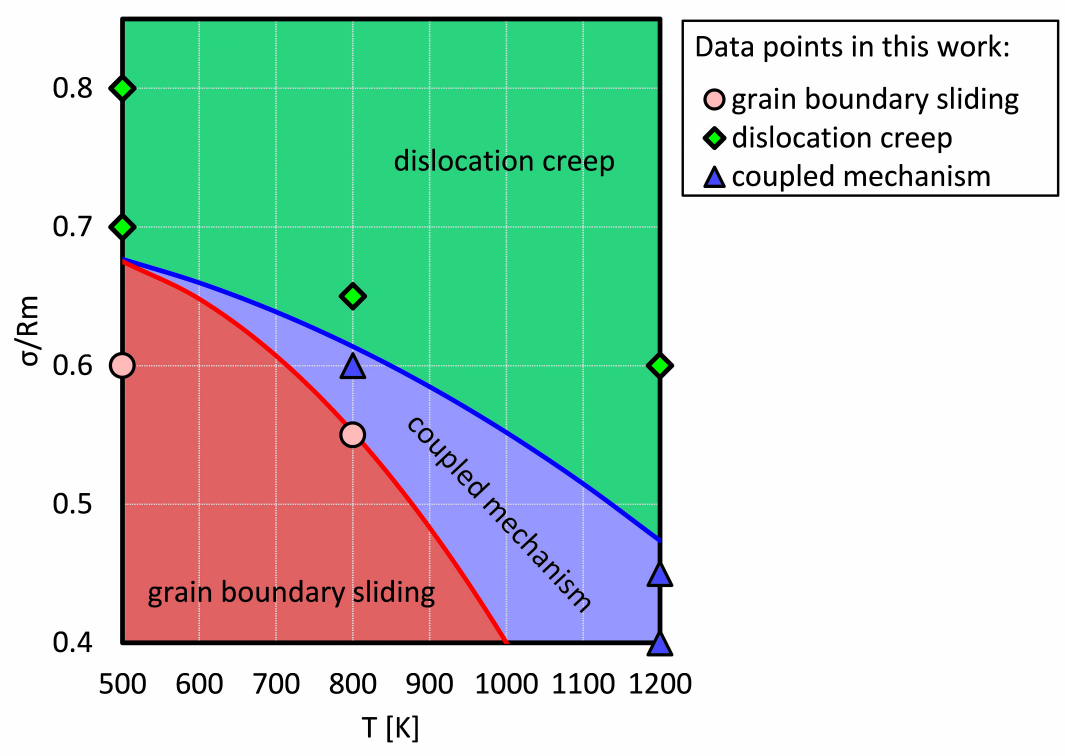

Figure 5. The schematic plastic deformation map created in this work. The applied stress was normalized to the tensile strength. The coupled mechanism means the creep mechanism is coupled by dislocation creep and grain boundary creep.

It is clear that at a low temperature and with a low stress, the creep process is dominated by grain boundary sliding, because the dislocation is difficult to nucleate at grain boundaries and to propagate into the grain. However, at a high stress level, dislocation nucleation and gliding is the dominant mechanism.

There is a common area (the blue area in Figure 5) where both dislocations and grain boundary sliding mechanisms become visible and make comparable contributions to the creep process. However, the red and green areas depict that a mechanism is dominant (but not exclusive). We propose that the transition from a grain boundary sliding dominated creep to a dislocation dominated creep is narrow at a lower temperature, e.g., the distance between the blue and the red line is smaller at $700 \mathrm{~K}$ than at $800 \mathrm{~K}$. The conjunction point at around $0.68 R_{\mathrm{m}}$ approximately represents that the transition between grain boundary sliding and dislocation creep is rarely observable.

However, for a creep process with a coupled mechanism, to accurately determine the contribution of each mechanism is rather difficult. Hence, the region of the coupled mechanism is to represent that the grain boundary sliding and dislocation creep possess a comparable contribution to a creep process, which will be discussed in Section 4.3.

Figure 6 is the famous Ashby map for Ni with a grain size of $32 \mu \mathrm{m}$ [16]. The area in the magenta box shows the region of interest with mechanisms found in this work. It is significant that our result is comparable to the Ashby map. In this work, the lowest normalized stress $\sigma / G=1.16 \times 10^{-2}$ for $0.4 R_{\mathrm{m}}$ at $1200 \mathrm{~K}$ is still higher than the stress corresponding to dislocation creep and grain boundary creep in the Ashby map. Because of the size effect, the yield strength and tensile strength are almost 7 times higher than normal results, e.g., $368 \mathrm{MPa}$ at $973.15 \mathrm{~K}$ [35]. This is presumed to be the reason why a comparable deformation diagram is obtained at a higher stress level.

As we have already discussed the tensile simulation in Section 3.1, the main parts of this section are dedicated to dislocation creep, grain boundary sliding, and their coupling. 


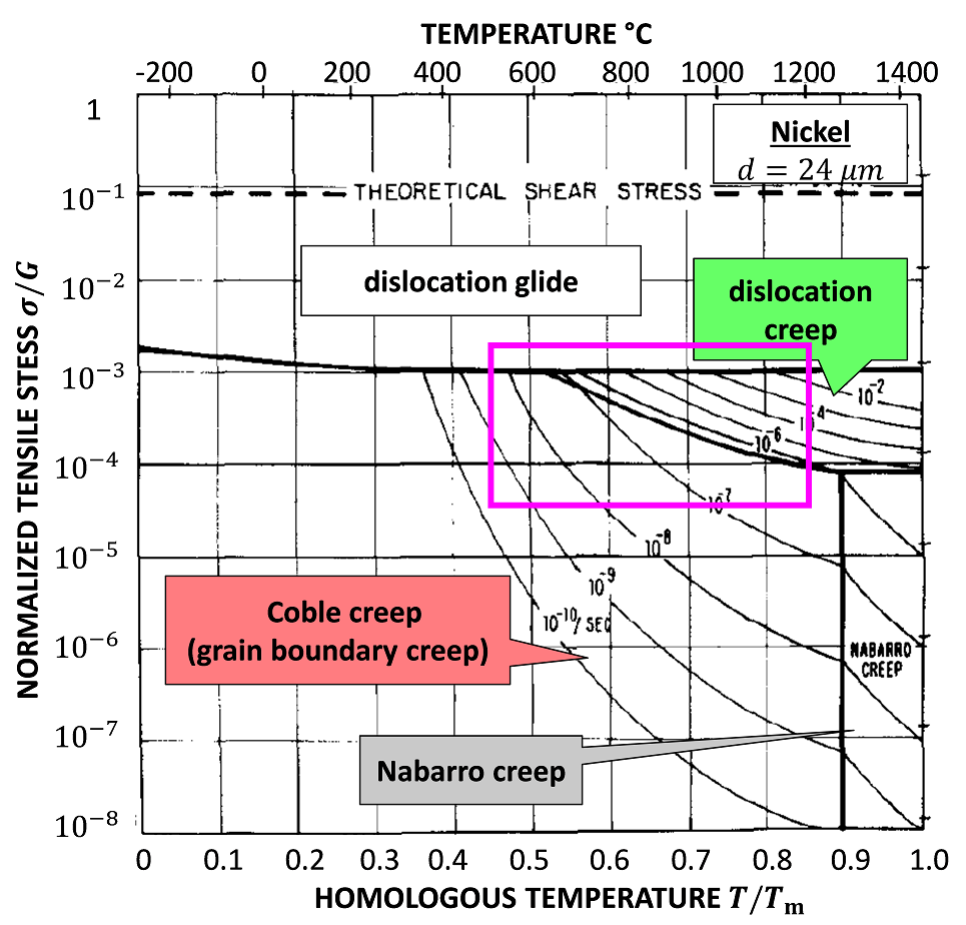

Figure 6. The Ashby map for plastic deformation of pure Ni with the grain size of $32 \mu \mathrm{m}$ [16]. The area in the magenta box coincides with this work as shown in Figure 5.

\subsection{Creep through Dislocations}

In Figure 7, snapshots of model M1 from different creep processes at the moment of $100 \mathrm{ps}$ are shown. It is significant that at a higher stress level (refer to the green area in Figure 5), many more dislocations and stacking faults appear. From Figure $7 \mathrm{c}$ for $1200 \mathrm{~K}$ and $0.4 R_{\mathrm{m}}$ and Figure $7 \mathrm{~d}$ for $1200 \mathrm{~K}$ and $0.65 R_{\mathrm{m}}$, intensive grain boundary sliding is also observed.

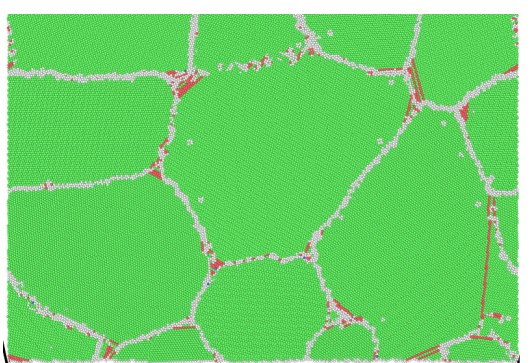

(a)

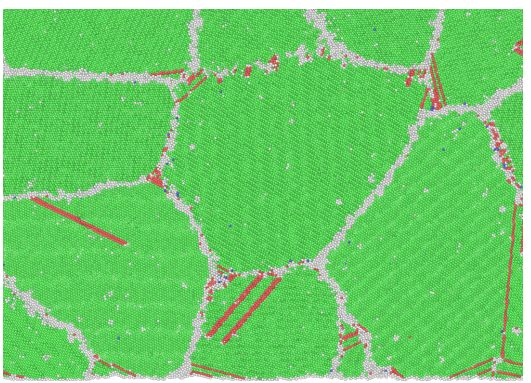

(c)

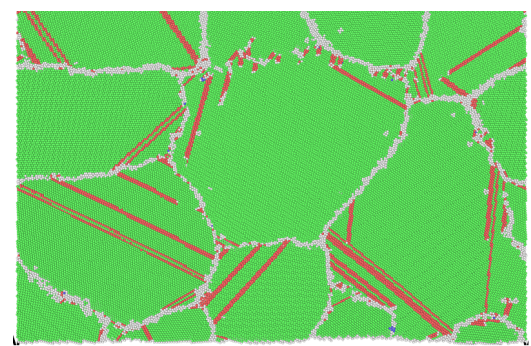

(b)

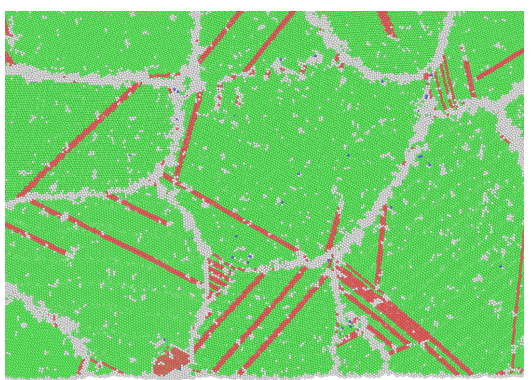

(d)

Figure 7. (a-d) Snapshots of atomic-scale crystalline structures of model M1 at 100 ps in different creep processes. (Green: FCC; red: stacking fault; gray: other.) (a) $500 \mathrm{~K}-0.4 R_{\mathrm{m}}-100 \mathrm{ps}$, (b) $500 \mathrm{~K}-0.8$ $R_{\mathrm{m}}-100 \mathrm{ps}$, (c) $1200 \mathrm{~K}-0.4 R_{\mathrm{m}}-100 \mathrm{ps}$, (d) $1200 \mathrm{~K}-0.65 R_{\mathrm{m}}-100 \mathrm{ps}$. 
Referring to Table 3, the calculated stress exponents $n$ for high stress are 13.8 and 6.8 for 500 and $1200 \mathrm{~K}$, respectively. Combined with Figure 7, it is in no doubt that the dislocation nucleation and propagation dominate the creep process at a high stress level.

Besides, through the dislocation extraction algorithm (DXA) [36] in OVITO, we observed dislocations interacting with stacking faults and also with other dislocations, as shown in Figure 8. For example, in Figure 8a, the moving dislocation was first blocked by a two-atom-layer stacking fault and then the dislocation went through the stacking fault with the glide plane jumping for one atom layer. Figure $8 \mathrm{~b}$ illustrates the dislocation meeting with a vacancy. One atom, the nearest neighbor to the vacancy, diffused into the dislocation half plane so that the dislocation line has a sharp peak at that point. However, the dislocation could not climb to the other glide plane because this peak vanished and the dislocation kept moving in the next configuration. This is probably because the mobility of the vacancy is still limited by the temperature.

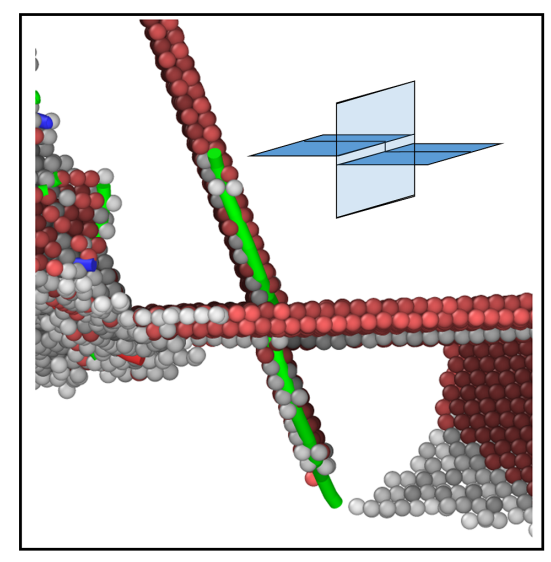

(a)

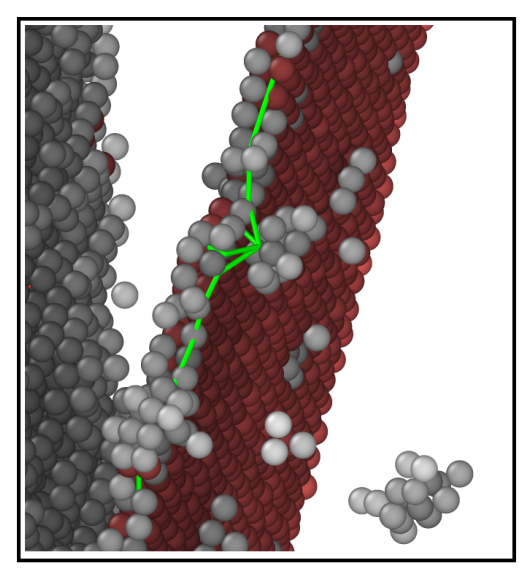

(b)

Figure 8. Dislocation activities observed in dislocation creep regime. (a) A dislocation jog as a dislocation moves through a stacking fault. (b) The half plane of the dislocation line has jumped into a vacancy. (Red atoms: stacking faults; gray atoms: other; green lines: Shockley partial dislocations.)

\subsection{Creep Through Grain Boundary Sliding}

The snapshots in Figure 9 show different moments of the creep process of model M1 at $1200 \mathrm{~K}$ and $0.4 R_{\mathrm{m}}$. FCC and HCP structures are presented in green and red, respectively. Other structures are shown in gray. Some of the gray clusters of other atoms inside grains are vacancies, which have been discussed in Section 3.3.

From Figure $9 \mathrm{a}-\mathrm{c}$, the boundaries of two grains, which can clearly be seen in the marked ellipses, were moving out of sight. At 100 ps, dislocation appears inside some grains (black circles in Figure 9b) and is elongated in the following snapshots. It is obvious that the dislocation nucleated at the grain boundary and then elongated inside the grain until it was impeded by other dislocations or the opposite grain boundary.

Additionally, two creep processes were compared with each other. Details are shown in Table 4 . The creep rate at $1200 \mathrm{~K}$ and $0.4 R_{\mathrm{m}}(0.90 \mathrm{GPa})$ is $2.26 \times 10^{7} 1 / \mathrm{s}$, which is comparable to the creep rate at $800 \mathrm{~K}$ and $0.65 R_{\mathrm{m}}(1.63 \mathrm{GPa})$ as $3.98 \times 10^{7} 1 / \mathrm{s}$, see Figure $3 \mathrm{~b}$. Whereas, stress exponents $n$ for the pairs $\left(1200 \mathrm{~K}, 0.4 R_{\mathrm{m}}\right)$ and $\left(800 \mathrm{~K}, 0.65 R_{\mathrm{m}}\right)$ as shown in Table 3 are obtained as 4.8 and 10.0, respectively. $n=10.0$ indicates that the mechanism is dislocation creep for simulation at $800 \mathrm{~K}$ and $0.65 R_{\mathrm{m}}$. However, with a similar creep rate, the amount of stacking faults of model M1 at $1200 \mathrm{~K}$ and $0.4 R_{\mathrm{m}}$ (2.4\% to all atoms) is much lower than that at $800 \mathrm{~K}$ and $0.65 R_{\mathrm{m}}(4.2 \%$ to all atoms). Therefore, it is convincing that the dominant creep mechanism for creep at lower stresses is grain boundary sliding. When the temperature increases and dislocations are thermally activated, then dislocation nucleation and propagation start to contribute. 


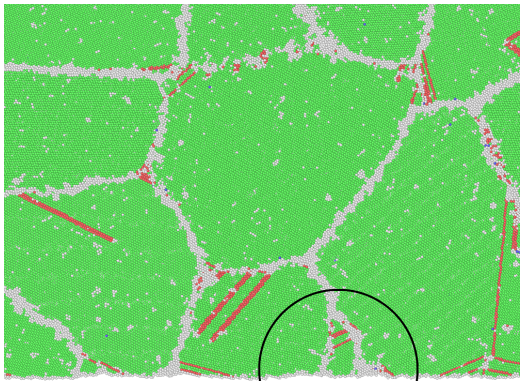

(a)

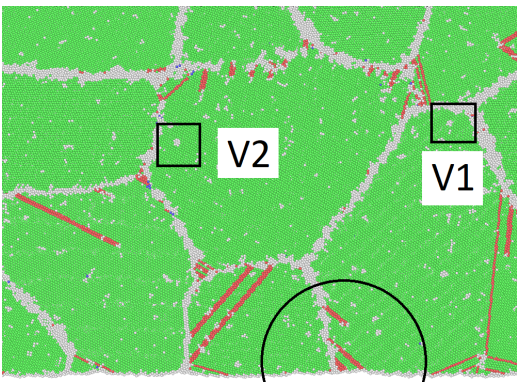

(c)

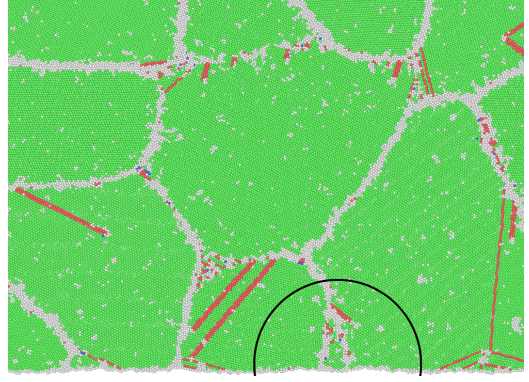

(b)

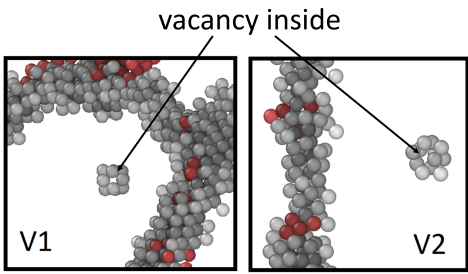

(d)

Figure 9. (a-c) Snapshots of atomic-scale crystalline structures of model M1 at $1200 \mathrm{~K}$ and $0.4 R_{\mathrm{m}}$ at different times in the creep process. The grain depicted in the half circle was moving out of sight during the creep process from 100 to 200 ps. (d) The nearest neighbor atoms of two vacancies that are enlarged from (c). For a clear illustration, the FCC atoms are deleted in (d). (Green: FCC; red: stacking fault; gray: others.) (a) 100 ps; (b) 150 ps; (c) 200 ps; (d) two vacancies.

Table 4. Comparison of two creep processes. The stacking fault ratio represents how many are atoms with an HCP structure compared with the total number of atoms in the model.

\begin{tabular}{ccc}
\hline Temperature [K] & 800 & 1200 \\
\hline stress level & $0.65 R_{\mathrm{m}}$ & $0.4 R_{\mathrm{m}}$ \\
\hline stress [GPa] & 1.76 & 0.90 \\
\hline creep rate $[1 / \mathrm{s}]$ & $3.98 \times 10^{7}$ & $2.26 \times 10^{7}$ \\
\hline stress exponent $n$ & 10.0 & 4.8 \\
\hline stacking fault ratio at $100 \mathrm{ps}$ & $4.3 \%$ & $2.4 \%$ \\
\hline
\end{tabular}

\subsection{Grain Size Effect}

The grain size is the third factor that influences the creep properties. According to Equation (1), the grain size exponent can be calculated as $n=\partial(\log \dot{\varepsilon}) / \partial(\log (1 / d))$. The grain size exponents $p$ of the five models are 2.56 and 2.57 , respectively, at the stress levels $0.7 R_{\mathrm{m}}$ and $0.8 R_{\mathrm{m}}$, see Figure 10. This means that, at a high stress level, the creep rate decreases with increasing grain size. Because of the limitation of grain size researched in previous works, dislocation nucleation was observed and analyzed. However, the interaction between dislocations inside the grain has not been studied in a nano-creep simulation. Around the transition regime between the Hall-Petch and inverse Hall-Petch effects, the grain size has a sufficient influence on the dislocation movement, which is closely related to the mechanical properties. Therefore, it is not trivial to investigate further in this direction. 


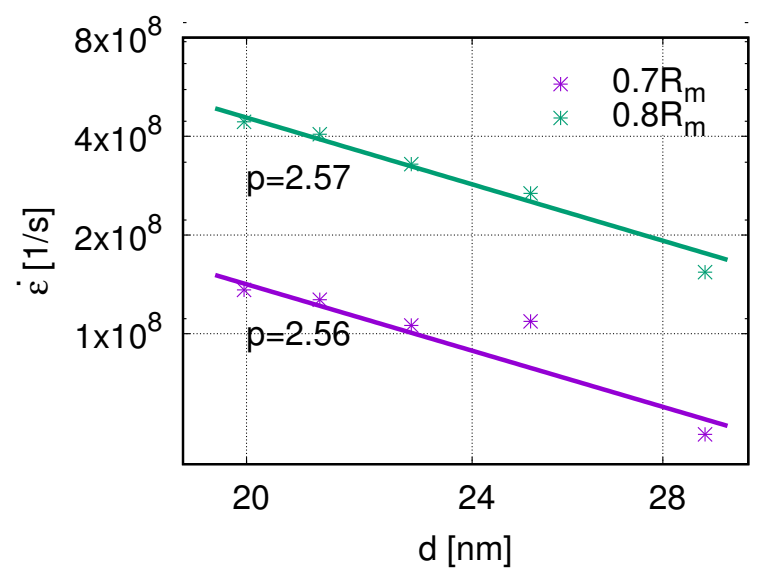

Figure 10. Log-log scaling plot of creep rate $\dot{\varepsilon}$ over grain size $d$ and the fit-curve at $800 \mathrm{~K}$. The grain size exponents are $p=2.57$ at $0.7 R_{\mathrm{m}}$ and $p=2.04$ at $0.8 R_{\mathrm{m}}$, respectively.

\section{Summary}

Through MD-simulations, we first studied the tensile properties of Ni nano-polycrystals. As the temperature decreased, the tensile strength $R_{\mathrm{m}}$ increased. The tensile strength showed no significant dependence on the grain size. This might be due to the fact that the investigated grain sizes lie in the transition area between the Hall-Petch and inverse Hall-Petch regimes.

With applying stress that was homologous to the tensile strength $R_{\mathrm{m}}$, nano-creep simulations were performed at different temperatures for all five models. From the creep curves, three typical creep phases have been clearly identified. We draw the conclusion that the creep rate rises with increasing stress, increasing temperature, and decreasing grain size. Collecting all plastic deformation cases, we formed a deformation mechanism map to distinguish the corresponding mechanisms at given conditions.

When applying a high stress, the stress exponent $n$ was above 6.7 , resulting in a dislocation creep. Visualization analysis revealed many dislocation movements, including nucleating from the grain boundary, propagating inside grains, and interacting with other dislocations or with grain boundaries.

As for a creep simulation at a low stress level, the dominant creep mechanism is supposed to be grain boundary sliding at a low temperature with stress exponents $n<3$. When increasing the temperature to $1200 \mathrm{~K}$, the stress exponent for the low stress part increases to 4.8 and dislocations begin to contribute to the creep process. Hence, it is safely concluded that the dominant creep mechanism is grain boundary sliding at low stress and this could be coupled with dislocation creep with increasing the temperature. Additionally, from the analysis of activation energies, it was found that the vacancy diffusion becomes prevalent when the temperature is above $1000 \mathrm{~K}$. The grain boundary creep is assumed to be enhanced by vacancy diffusion at high temperatures. Furthermore, we postulate that the creep mechanism of NC Ni remains unchanged with the coupling of dislocation creep and grain boundary creep when the temperature is above $1400 \mathrm{~K}$.

It is novel that the deformation diagram corresponds well to the Ashby map for pure Ni. Due to the properties of NC metals, it is difficult and expensive to experimentally investigate the creep behavior of NC metals at high temperatures. This work could serve as a good example to expand deformation diagrams for NC metals through employing molecular dynamics simulations.

Author Contributions: Conceptualization, S.S. and P.B.; methodology, P.B., W.V., and S.S.; software, X.X., P.B., and W.V.; formal analysis, X.X.; investigation, X.X. and W.V.; writing-original draft preparation, X.X.; writing-review and editing, X.X., P.B., W.V., and S.S.; visualization, X.X.; supervision, S.S. and P.B. All authors have read and agreed to the published version of the manuscript. 
Funding: We thank the Deutsche Forschungsgemeinschaft (DFG, German Research Foundation) for supporting this work by funding-EXC2075-390740016 under Germany's Excellence Strategy. We acknowledge the support by the Stuttgart Center for Simulation Science (SimTech).

Data Availability Statement: The data presented in this study are available on request from the corresponding author. The data are not publicly available due to the ongoing research.

Acknowledgments: The authors acknowledge support by the state of Baden-Württemberg through bwHPC.

Conflicts of Interest: The authors declare no conflict of interest.

\section{References}

1. Hahn, E.N.; Meyers, M.A. Grain-size dependent mechanical behavior of nanocrystalline metals. Mater. Sci. Eng. A 2015, 646, 101-134. [CrossRef]

2. Wu, Z.; Sandlöbes, S.; Wang, Y.; Gibson, J.S.L.; Korte-Kerzel, S. Creep behaviour of eutectic Zn-Al-Cu-Mg alloys. Mater. Sci. Eng. A 2018, 724, 80-94. [CrossRef]

3. Wu, J.; Tsai, W.; Huang, J.; Hsieh, C.; Huang, G.R. Sample size and orientation effects of single crystal aluminum. Mater. Sci. Eng. A 2016, 662, 296-302. [CrossRef]

4. Carlton, C.; Ferreira, P. What is behind the inverse Hall-Petch effect in nanocrystalline materials? Acta Mater. 2007, 55, 3749-3756. [CrossRef]

5. Mukherjee, A.K.; Bird, J.E.; Dorn, J.E. Experimental Correlations for High-Temperature Creep; Technical Report; University of California: Berkeley, CA, USA, 1968.

6. Coble, R. A model for boundary diffusion controlled creep in polycrystalline materials. J. Appl. Phys. 1963, 34, 1679-1682. [CrossRef]

7. Cahn, R.; Haasen, P.; Kramer, E. Plastic Deformation and Fracture of Materials. In Materials Science and Technology; Mughrabi, H., Ed.; Volume 6; WILEY-VCH Verlag GmbH \& Co. KGaA: Weinheim, Germany, 1993.

8. Ashby, M. Boundary defects, and atomistic aspects of boundary sliding and diffusional creep. Surf. Sci. 1972, 31, 498-542. [CrossRef]

9. Keblinski, P.; Wolf, D.; Gleiter, H. Molecular-dynamics simulation of grain-boundary diffusion creep. Interface Sci. 1998, 6, 205-212. [CrossRef]

10. Herring, C. Diffusional viscosity of a polycrystalline solid. J. Appl. Phys. 1950, 21, 437-445. [CrossRef]

11. Berry, J.; Rottler, J.; Sinclair, C.W.; Provatas, N. Atomistic study of diffusion-mediated plasticity and creep using phase field crystal methods. Phys. Rev. B 2015, 92, 134103. [CrossRef]

12. Nie, K.; Wu, W.P.; Zhang, X.L.; Yang, S.M. Molecular dynamics study on the grain size, temperature, and stress dependence of creep behavior in nanocrystalline nickel. J. Mater. Sci. 2017, 52, 2180-2191. [CrossRef]

13. Jiao, S.; Kulkarni, Y. Molecular dynamics study of creep mechanisms in nanotwinned metals. Comput. Mater. Sci. 2015, 110, 254-260. [CrossRef]

14. Wang, Y.J.; Ishii, A.; Ogata, S. Transition of creep mechanism in nanocrystalline metals. Phys. Rev. B 2011, 84, 224102. [CrossRef]

15. Wang, Y.J.; Ishii, A.; Ogata, S. Grain size dependence of creep in nanocrystalline copper by molecular dynamics. Mater. Trans. 2012, 53, 1110171491. [CrossRef]

16. Ashby, M.F. A first report on deformation-mechanism maps. Acta Metall. 1972, 20, 887-897. [CrossRef]

17. Van Swygenhoven, H.; Caro, A. Plastic behavior of nanophase Ni: A molecular dynamics computer simulation. Appl. Phys. Lett. 1997, 71, 1652-1654. [CrossRef]

18. Van Swygenhoven, H.; Caro, A. Plastic behavior of nanophase metals studied by molecular dynamics. Phys. Rev. B 1998, 58, 11246. [CrossRef]

19. Reddy, K.V.; Meraj, M.; Pal, S. Mechanistic study of bending creep behaviour of bicrystal nanobeam. Comput. Mater. Sci. 2017, 136, 36-43. [CrossRef]

20. Meraj, M.; Yedla, N.; Pal, S. The effect of porosity and void on creep behavior of ultra-fine grained nano crystalline nickel. Mater. Lett. 2016, 169, 265-268. [CrossRef]

21. Yamakov, V.; Wolf, D.; Phillpot, S.; Mukherjee, A.; Gleiter, H. Deformation-mechanism map for nanocrystalline metals by molecular-dynamics simulation. Nat. Mater. 2004, 3, 43-47. [CrossRef]

22. Yamakov, V.; Wolf, D.; Salazar, M.; Phillpot, S.; Gleiter, H. Length-scale effects in the nucleation of extended dislocations in nanocrystalline Al by molecular-dynamics simulation. Acta Mater. 2001, 49, 2713-2722. [CrossRef]

23. Yamakov, V.; Wolf, D.; Phillpot, S.R.; Mukherjee, A.K.; Gleiter, H. Dislocation processes in the deformation of nanocrystalline aluminium by molecular-dynamics simulation. Nat. Mater. 2002, 1, 45-49. [CrossRef] [PubMed]

24. Yamakov, V.; Wolf, D.; Phillpot, S.; Gleiter, H. Grain-boundary diffusion creep in nanocrystalline palladium by moleculardynamics simulation. Acta Mater. 2002, 50, 61-73. [CrossRef]

25. Haslam, A.; Yamakov, V.; Moldovan, D.; Wolf, D.; Phillpot, S.; Gleiter, H. Effects of grain growth on grain-boundary diffusion creep by molecular-dynamics simulation. Acta Mater. 2004, 52, 1971-1987. [CrossRef] 
26. Pal, S.; Meraj, M.; Deng, C. Effect of Zr addition on creep properties of ultra-fine grained nanocrystalline Ni studied by molecular dynamics simulations. Comput. Mater. Sci. 2017, 126, 382-392. [CrossRef]

27. Pinky, S.K. Molecular Dynamics Study of Creep Deformation in Nickel-Based Superalloy. Master's Thesis, Missouri State University, Springfield, MO, USA, 2019.

28. Hirel, P. Atomsk: A tool for manipulating and converting atomic data files. Comput. Phys. Commun. 2015, 197, 212-219. [CrossRef]

29. Plimpton, S. Fast parallel algorithms for short-range molecular dynamics. J. Comput. Phys. 1995, 117, 1-19. [CrossRef]

30. Bonny, G.; Pasianot, R.C.; Castin, N.; Malerba, L. Ternary Fe-Cu-Ni many-body potential to model reactor pressure vessel steels: First validation by simulated thermal annealing. Philos. Mag. 2009, 89, 3531-3546. [CrossRef]

31. Voter, A.F.; Chen, S.P. Accurate interatomic potentials for Ni, Al and Ni3Al. MRS Online Proc. Libr. OPL 1986, 82, 175. [CrossRef]

32. Stukowski, A. Visualization and analysis of atomistic simulation data with OVITO-the Open Visualization Tool. Model. Simul. Mater. Sci. Eng. 2009, 18, 015012. [CrossRef]

33. Larsen, P.M.; Schmidt, S.; Schiøtz, J. Robust structural identification via polyhedral template matching. Model. Simul. Mater. Sci. Eng. 2016, 24, 055007. [CrossRef]

34. Aidhy, D.S.; Lu, C.; Jin, K.; Bei, H.; Zhang, Y.; Wang, L.; Weber, W.J. Point defect evolution in Ni, NiFe and NiCr alloys from atomistic simulations and irradiation experiments. Acta Mater. 2015, 99, 69-76. [CrossRef]

35. Anand, T.J.S. Nickel as an alternative automotive body material. J. Mech. Eng. 2012, 2, 187-197.

36. Stukowski, A.; Bulatov, V.V.; Arsenlis, A. Automated identification and indexing of dislocations in crystal interfaces. Model. Simul. Mater. Sci. Eng. 2012, 20, 085007. [CrossRef] 